Research Paper

\title{
SSRP1 influences colorectal cancer cell growth and apoptosis via the AKT pathway
}

\author{
Qian Wang ${ }^{1}$, Shengnan Jia ${ }^{2}$, Yan Jiao ${ }^{3}$, Libo Xu ${ }^{1}$, Ding Wang ${ }^{1}$, Xuyang Chen ${ }^{1}$, Xindan $\mathrm{Hu}^{1}$, Hang Liang ${ }^{1}$, \\ Naiyan Wen ${ }^{1}$, Shengnan Zhang ${ }^{1}$, Baofeng Guo ${ }^{\circledR}$, Ling Zhang ${ }^{\circledR}$ \\ 1. Department of Pathophysiology, College of Basic Medical Science, Jilin University, Changchun 130021, P. R. China; \\ 2. Department of Hepatopancreatobiliary Medicine, The Second Hospital of Jilin University, Changchun 130041, P. R. China; \\ 3. Department of Hepatobiliary and pancreatic surgery, First Hospital of Jilin University, Changchun 130021, P. R. China; \\ 4. Department of Plastic Surgery, China-Japan Union Hospital, Jilin University, Changchun 130033, P. R.China. \\ $\square$ Corresponding authors: Dr Ling Zhang, Department of Pathophysiology, College of Basic Medical Science, Jilin University, 126 Xinmin Street, Changchun, \\ Jilin 130021, P.R. China, E-mail: zhangling3@jlu.edu.cn and Dr. Baofeng Guo, Department of Plastic Surgery, China-Japan Union Hospital, Jilin University, \\ Changchun 130033, P. R.China, E-mail: gbf_cc@sohu.com
}

(c) The author(s). This is an open access article distributed under the terms of the Creative Commons Attribution License (https://creativecommons.org/licenses/by/4.0/). See http:/ /ivyspring.com/terms for full terms and conditions.

Received: 2019.07.15; Accepted: 2019.09.11; Published: 2019.10.21

\begin{abstract}
Colorectal cancer is one of the most common cancers worldwide with a high incidence rate. Therefore, the molecular basis of colorectal tumorigenesis and evolution must be clarified. Structure-specific recognition protein 1 (SSRPI) is involved in transcriptional regulation, DNA damage repair, and cell cycle regulation and has been confirmed to be highly expressed in various tumor tissues, including colorectal cancer. However, the role of SSRPI in the development of colorectal cancer remains unclear. Therefore, this study explored the role of SSRPI in the occurrence and development of colorectal cancer. Using bioinformatics databases, including samples from the Cancer Genome Atlas (TCGA), we confirmed high SSRPI expression in human colorectal adenocarcinoma tissues. We demonstrated that SSRPI knockdown via small interfering RNA significantly inhibited the proliferation of colorectal cancer cells and promoted apoptosis through the AKT signaling pathway, suppressing the invasion and migration of colorectal cancer cells in vitro and in vivo. In conclusion, this study demonstrated that SSRPI silencing influenced the proliferation and apoptosis of colorectal cancer cells via the AKT signaling pathway.
\end{abstract}

Key words: SSRP1, proliferation, apoptosis, AKT pathway

\section{Introduction}

Colorectal cancer is one of the most common malignant tumors of the digestive system and is the second-ranked tumor for cancer deaths globally, with an increase of more than 1 million new cases annually 1, 2. Radiotherapy, chemotherapy, molecular-targeted therapy, and palliative surgery are current treatment options for patients with colorectal tumor metastases; however, the 5-year survival rate remains less than $10 \% 3,4$. Therefore, further research on the pathogenesis of colorectal cancer is of great significance for preventing its occurrence and improving prognosis.

Structural-specific recognition protein 1 (SSRP1) is involved in transcriptional regulation, DNA damage repair, and cell cycle regulation ${ }^{5}$. SSRP1 is highly expressed in a variety of tumor tissues, while it is lowly expressed in normal mature tissues ${ }^{6}$. Curaxins, compounds with activity against SSRP1, have been shown to induce apoptosis in tumor cells ${ }^{7}$. Downregulation of SSRP1 by siRNA technology can also inhibit the proliferation of U87 and U251 glioma cells through the MAPK pathway ${ }^{8}$. SSRP1 is suggested to play a role in the occurrence and development of tumors and thus provides a large platform for further studying the mechanisms of colorectal cancer development.

In human colorectal cancer, whether SSRP1 plays a critic role and its underlying mechanisms of tumor genesis and evolution is unclear. To clarify it, we first analyze the SSRP1 expression by TCGA 
databases and cell lines, and identify its influence on cell proliferation and apoptosis by AKT pathway. Besides, in vitro and in vivo experiments also prove its migration and invasion influence. Therefore, SSRP1 may providesa possible therapeutic strategy and diagnostic targets on human colorectal cancer in clinic.

\section{Materials and methods}

\section{Bioinformatics}

GEPIA (http://gepia.cancer-pku.cn/index.html) and UALCAN browser (http://ualcan.path.uab.edu/index. $h t m l)$, developed interactive web servers, were used for analyzing data of samples from the TCGA.

\section{Cell culture}

Human colorectal cancer cell lines including DLD1, SW620, HCT15, HCT116, HCT8 and a normal human colon mucosal epithelial cell line NCM460 were gifted by the School of Public Health, Jilin University (Jilin, China). The cell lines were all cultured in RPMI 1640 medium (Gibco, Thermo Fisher Scientifc, Inc., Waltham, MA, USA), supplemented with $10 \%$ foetal bovine serum (FBS, BI, Israel), $100 \mathrm{U} / \mathrm{mL}$ penicillin and $100 \mathrm{mg} / \mathrm{mL}$ streptomycin (Sigma, St. Louis, MO, USA). Cells were cultured at $37^{\circ} \mathrm{C}$ in a humidified atmosphere containing $5 \% \mathrm{CO}_{2}$.

\section{Transient transfection}

SW620 and HCT15 cells were transfected with siRNAs, which were purchased from Genepharma (Suzhou, China) and the sequences of siRNAs were as follows: siSSRP1-1 sense, 5'-GCCAUGUCUACAAGU AUGATT-3' and antisense, 5'-UCAUACUUGUAGAC AUGGCTT-3'; siSSRP1-2 sense, 5'-CCCAGAAUGGU GUUGUCAAATT-3' and antisense, 5'-UUUGACAAC ACAUUCUGGGTT-3'; negative control sense, 5'-CAC GCAGAACGTGAACACC-3' and antisense, 5'-GGCA GTAGATAACGTGAGGGA-3'. Prior to transfection, cells were cultured in 96-well plates or 6-well plates ensuring that these cells had reached $80 \%$ confluency the next day. The thermo transfection agent Interferin (Thermo Fisher Scientific, Inc., Waltham, MA, USA) was used according to the manufacturer's protocol. Cells were collected after transfection at 48-72 h.

\section{Cell viability, proliferation and colony formation assay}

SW620 and HCT15 cells were plated onto 96-well plates at a concentration of $1 \times 10^{4}$ cells/well to detect cell viability using Cell Counting Kit-8 (CCK-8; MCE, New Jersey, USA) at $24 \mathrm{~h}, 48 \mathrm{~h}$ and $72 \mathrm{~h}$ after transfection. The OD450 was measured by FLUO star Omega reader (BMG LABTECH, Ortenberg,
Germany). Cells were seeded on 6-well plates after transfection at a concentration of $5 \times 10^{4}$ cells/well and cell growth was assessed by growth counting at $24 \mathrm{~h}$, $48 \mathrm{~h}$ and $72 \mathrm{~h}$. For colony formation assay, 100 cells/well were plated on 6-well plates and in 5-7 days after transfection the colonies were fixed with $4 \%$ paraformaldehyde and then stained with $0.2 \%$ crystal violet. The number of colony formation was measured by IX71 inverted fluorescence microscope (Olympus, Shinjuku, Tokyo, Japan).

\section{Cell cycle analysis}

Cells were collected by trypsinization and washed twice with cold PBS. After fixation in ice-cold $75 \%$ ethanol for one hour at $-20^{\circ} \mathrm{C}$ or overnight at $4{ }^{\circ} \mathrm{C}$, cells were washed with cold PBS and resuspended with $500 \mu \mathrm{L}$ cold PBS. Then $20 \mu \mathrm{L}$ RNase A solution was added and incubated in a $37^{\circ} \mathrm{C}$ water bath for 30 minutes. Each sample was stained with $400 \mu \mathrm{L}$ PI stain solution (BestBio, ShangHai, China) at $4^{\circ} \mathrm{C}$ for 60 $\mathrm{min}$ in the dark. Cell cycle distributions were detected by Accuri C6 flow cytometer (Becton Dickinson, Franklin Lakes, New Jersey, USA).

\section{Apoptosis analysis}

SW620 and HCT15 cells were plated onto 6-well plates at a concentration of $1 \times 10^{5}$ cells/well. Transfection experiments were performed on the following day. SH-6 (10uM), an inhibitor of AKT, was obtained from Abcam. Cells were harvested in $48 \mathrm{~h}$ and washed twice with cold PBS. Subsequently cells were resuspended and stained with $5 \mu \mathrm{L}$ Annexin-V-FITC and $5 \mu \mathrm{L}$ PI $(50 \mu \mathrm{g} / \mathrm{mL})$ of Annexin V FITC Apop Dtec Kit (BD Biosciences, San Jose, CA, USA) in the dark at room temperature for $15 \mathrm{~min}$ and detected by Accuri C6 flow cytometer (Becton Dickinson, Franklin Lakes, New Jersey, USA).

\section{Cell migration and invasion assays}

Cell migration assay was conducted using Transwell chambers (Coastor, Corning, USA) which were 8-mm pore size. The lower chamber was filled with RPMI 1640 medium containing 20\% FBS (BI, Israel). However, the upper chamber was plated cells resuspended in serum-free DMEM and the concentration of every well was $5 \times 10^{4}$ cells. After cultivation in $5 \% \mathrm{CO}_{2}$ at $37^{\circ} \mathrm{C}$ for 48 hours, the bottom surface of the polycarbonate membranes in the upper chamber were wiped with cotton swabs to remove residual cells and cells were counted visually using IX71 inverted fluorescence microscope (Olympus, Shinjuku, Tokyo, Japan) after stained with $0.1 \%$ crystal violet dye. In contrast, matrigel (BD Biosciences, San Jose, CA, USA) was used in the transwell chambers (Coastor, Corning, USA) before cells were plated in the invasion assay. Cell migration 
and invasion were determined by counting five random fields under an optical microscope and the data are presented as mean \pm standard deviation (SD).

\section{Western blot analysis}

Cells and tissues were harvested and lysed in RIPA buffer for $30 \mathrm{~min}$ at $4-8^{\circ} \mathrm{C}$. Proteins were detected and quantified using BCA protein assay kit (Thermo Fisher Scientific). Protein samples (30-50 $\mu \mathrm{g})$ were separated by $12 \%$ SDS-PAGE and transferred onto polyvinylidene fluoride membranes. Nonspecific binding sites were blocked with 5\% low-fat milk and $0.1 \%$ Tween-20 at room temperature for $2 \mathrm{~h}$. Subsequently, the membranes were incubated overnight at $4^{\circ} \mathrm{C}$ with the following primary antibodies: Proteintech (Wuhan, China): anti-SSRP1 (1:1000; 15696-1-AP), anti-p27 (1:1000; 25614-1-AP), anti-p21 (1:1000; 10355-1-AP), anti-CyclinD1 (1:1000; 60186-1-Ig), anti-BCL2 (1:1000; 26593-1-AP), anti-BAX (1:1000; 50599-2-Ig), anti-MDM2 (1:1000; 19058-1-AP), anti-p53 (1:1000; 10442-1-AP), anti-AKT (1:1000; 60203-2-Ig), anti- $\beta$-actin (1:2000; 66009-1-Ig) and arigo (Taiwan, China): anti-P-AKT (1:1000; ARG51559); secondary antibody: goat anti-Mouse (1:2000; 10828-I-AP) and goat anti-Rabbit IgG (H+L) (1:2000; SA0000I-2) from Proteintech Group Inc. The membranes were scanned for statistical analysis by enhanced chemiluminescence (ECL) using a gel image processing system (Tanon, Shanghai) and the density of the bands was analyzed using Image J ( Wayne Rasband National Institutes of Health, USA).

\section{Animal experiments}

The siRNA used in vivo was synthesized by Genepharma (Suzhou, China) and dissolved in PBS buffer. The dose of siRNA in nude mice was $0.5 \mathrm{mg} / \mathrm{kg}$.

$10 \mathrm{BALB} / \mathrm{c}$ male nude mice aged $4-5$ weeks (male, 18-22 g) were purchased from Beijing Vital River Laboratory Animal Technology and housed under a 12/12 hour light/dark cycle in an air-conditioned room at $22 \pm 2{ }^{\circ} \mathrm{C}$ with free food and water. All animal experiments were undertaken in accordance with the National Institute of Health Guide for the Care and Use of Laboratory Animals, with the approval of the Scientific Investigation Board of the College of Basic Medicine, Jilin University. The nude mice were randomly divided into two groups. Each of them was received $100 \mu \mathrm{L}$ subcutaneous injection containing $5 \times 10^{5}$ HCT15 cells. When the tumor size reached to 3-5 mm, siSSRP1 or NC (isodose PBS) was inoculated into the xenograft tumor by multi-point injection three times a week. Tumor size was measured every 3 days with a Vernier caliper and tumor volume was calculated with the following formula: $\mathrm{V}=$ (length) $\times(\text { width })^{2} / 2$. After 34 days, mice were sacrificed by excessive intraperitoneal injection of barbiturates (Pentobarbital; $150 \mathrm{mg} / \mathrm{kg}$; Spofa, Prague) followed by cervical dislocation. Tumor tissues were resected to be frozen at $-80^{\circ} \mathrm{C}$ for protein assay or fixed with $4 \%$ paraformaldehyde for hematoxylin-eosin (HE) staining and immunofluorescent staining.

\section{TUNEL assay}

Tissue sections were treated by using One Step TUNEL Apoptosis Assay Kit (Beyotime Biotechnology Inc., Nantong, China) principally according to the instructions. TUNEL specimens were observed under the BX53 fluorescence microscope (Olympus, Japan) with a laser excitation at $488 \mathrm{~nm}$ to detect the FITC-labeled TUNEL-positive cells.

\section{Hematoxylin and eosin (H\&E) staining and Immunohistochemistry}

Tissues were fixed with $4 \%$ paraformaldehyde solution for at least $4 \mathrm{~h}$ at room temperature, followed by dehydration, dipping in wax, paraffin embedding and cut into sections. Then, these sections were treated with HE staining. For Immunohistochemistry, sections were incubated with serum or BSA for $30 \mathrm{~min}$ at room temperature, and then were dipped in diluted primary antibody for $2 \mathrm{~h}$ and then incubated with secondary antibody. The antibodies used in this research: primary antibodies: Proteintech (Wuhan, China): anti-SSRP1 (1:200; 15696-1-AP), anti-BCL2 (1:200; 26593-1-AP), anti-BAX (1:200; 50599-2-Ig), anti-MMP2 (1:200; 10373-2-AP), anti-MMP9 (1:200; 10375-2-AP) and PCNA (1:50; SC-56) from Santa Cruz Biotechnology; secondary antibody: goat anti-Rabbit IgG (H+L) (1:200; SA0000I-2) from Proteintech Group Inc. The sample was observed under BX53 fluorescence microscope (Olympus, Japan). Cells stained brown were positive cells.

\section{Statistical analysis}

All analyses were performed using Microsoft Excel or Prism GraphPad 6.00. Data analyses were performed from at least three independent experimental groups. Comparison of the two sets of data was performed using the unpaired Student's t-test. To compare more than two sets, one-way analysis of variance analysis (ANOVA) with a Newman-Keuls multiple comparison test was conducted. For all experiments with error bars, the standard deviation was calculated to indicate the variation within each experiment. Values represent mean \pm SEM. Differences were considered to be significant at ${ }^{*} p<0.05,{ }^{* *} p<0.01$, vs. NC group. 


\section{Results}

\section{SSRPI expression was upregulated in both human colorectal cancer tissues and cells}

Analysis of the SSRP1 expression in human tumor tissues from the Firehose Broad GDAC database (https://gdac.broadinstitute.org/\#) showed that SSRP1 is upregulated in multiple tumor tissue types (Fig. 1A). Data from the Gene Expression Profiling Interactive Analysis (GEPIA; http://gepia. cancer-pku.cn/) also showed that the mean expression level of SSRP1 in colorectal adenocarcinoma tissues was upregulated compared with the corresponding normal tissues $(P<0.01)$ (Fig. 1B). Results from the UALCAN database (http://ualcan. path.uab.edu/) also showed a higher level of SSRP1 in patients with colorectal adenocarcinoma cancer than in normal patients $(P<0.001)$ (Fig. 1C). Furthermore, we compared a normal colorectal cell

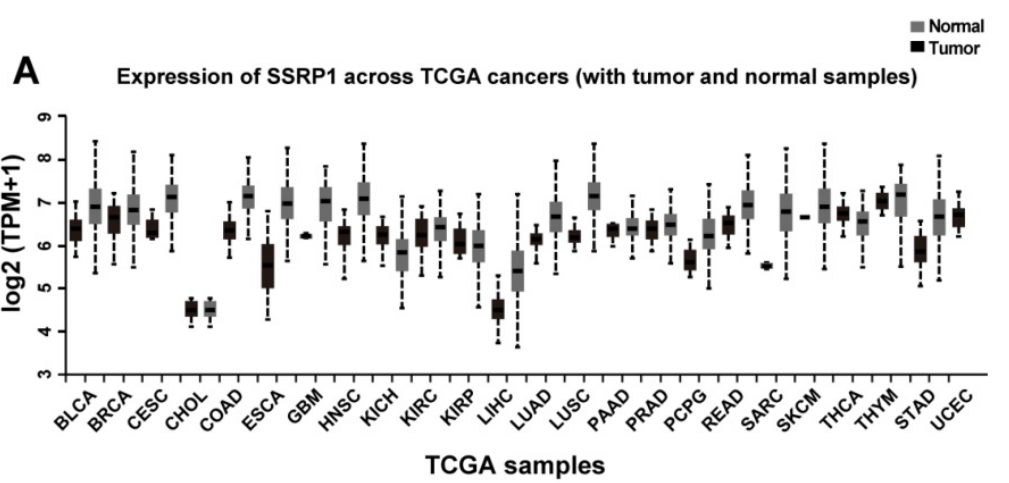

B

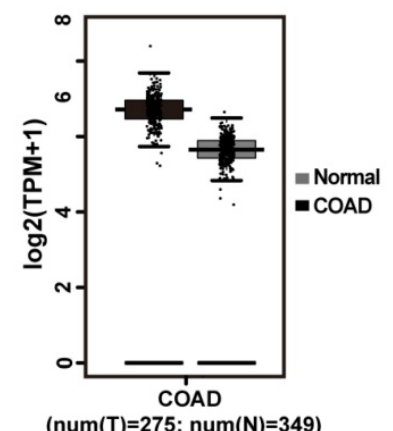

D

C

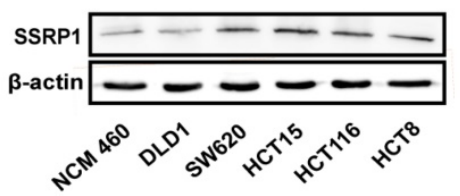

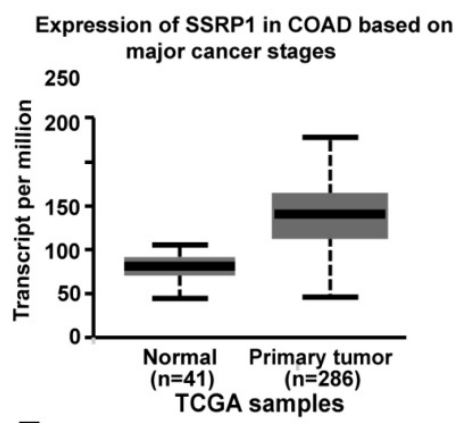

E

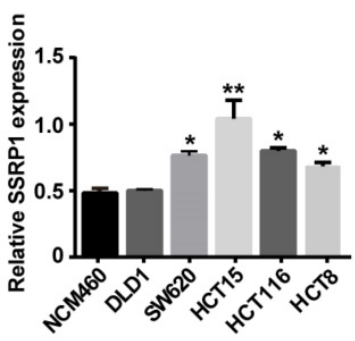

Figure 1. SSRPI expression is upregulated in both human colorectal cancer tissues and cells. (A) UALCAN database displays SSRP1 is in a high expression status in multiple tumor types. (B) GEPIA database shows the expression of SSRPI is up regulated conspicuously in colorectal cancer tissues $(n=275)$ versus normal tissues $(n=349)$. $(P<0.01)(C)$ UALCAN database shows an obvious higher expression of SSRP1 in colorectal cancer patients $(n=286)$ than normal patients $(n=41)$ from TCGA. $(P<0.01)(D, E)$ Protein levels of SSRPI in normal gastric cell line (NCM460) with a panel of colorectal cancer cell lines (DLD1, SW620, HCT15, HCT116, and HCT8). line (NCM460) with a panel of colorectal cancer cell lines (DLD1, SW620, HCT15, HCT116, and HCT8) for SSRP1 expression and found that SSRP1 was increased in the colorectal cancer cell lines (Figs. 1D, E). Based on the statistical data, we chose the HCT15 and SW620 cell lines for subsequent experiments.

\section{SSRPI inhibition repressed colorectal cancer cell proliferation}

To explore the function of SSRP1 in the development of colorectal cancer, we used RNAi technology to silence the expression of SSRP1 in colorectal cancer cells to observe whether SSRP1 modulation affected colorectal cancer cells. After siRNA transfection for $48 \mathrm{~h}$, SSRP1 protein levels in HCT15 and SW620 cells were downregulated, as assessed by western blotting (Fig. 2A, B). Supplementary Fig. S1A and B provide the data on the SSRP1 silencing in HCT116 cells.

We next examined the effects of SSRP1 on HCT15 and SW620 cell proliferation. Compared with cells transfected with the negative control (NC), the HCT15, SW620, and HCT116 cell viabilities at OD $450 \mathrm{~nm}$ decreased by different degrees after siRNA transfection for 24, 48 and $72 \mathrm{~h}$ (Fig. 2C, Supplementary Fig. S1C). Cell proliferation was also assessed using cell counting and colony formation assays. As expected, the cell number and colony formation ability of the HCT15 and SW620 cells decreased significantly after SSRP1 siRNA transfection (Fig. 2D, E, F). HCT116 cells treated with siRNA also exhibited weakened colony formation ability (Supplementary Fig. S1D, E, F). These data revealed that SSRP1 repressed the proliferation of colorectal cancer cells.

\section{SSRP 1 affected proliferation and apoptosis of colorectal cancer cells by inhibiting the AKT signaling pathway}

Based on the observed effects of SSRP1 on the proliferation of colorectal cancer cells, we examined the cell cycle and apoptosis of colorectal cancer cells by flow cytometry to further explore SSRP1 regulation of colorectal cancer cell proliferation. HCT15 and SE620 cells treated with siRNA for $48 \mathrm{~h}$ underwent significant G1 phase arrest compared with cells treated with NC (Fig. 3A, Supplementary Fig. S2A). The percentage of apoptotic cells in the siRNA-treated group was significantly increased (Fig. 3B, C). 
The AKT pathway is a classic signaling pathway involved in proliferation and apoptosis. To determine how SSRP1 regulates the cell cycle and apoptosis, we used western blotting to detect alterations in critical proteins in the AKT signaling pathway. Figure 3D shows that SSRP1 knockdown reduced AKT phosphorylation (P-AKT) and altered the cell cycle regulators, p21, p27 and cyclin D1 (Fig. 3D, Supplementary Fig. S2B). Furthermore, we found that downstream BAX expression was increased and BCL2 expression was decreased (Fig. 3E, Supplementary Fig. S2C). Significant increase of apoptotic rate and apoptosis-related protein in HCT15 cells treated with SSRP1 silence and SH-6 using western blot and flow cytometry (Figure 3F-I), illustrating the inhibition of
AKT signaling pathway indeed could induce apoptosis in colorectal cancer cells.

Therefore, SSRP1 silencing may activate the AKT signaling pathway to block proliferation and promote apoptosis.

\section{Downregulation of SSRPI inhibited migration and invasion of colorectal cancer cells}

Our study also verified that SSRP1 affected migration and invasion of colorectal cancer cells. Transwell assays showed that downregulation of SSRP1 using siRNA impaired the migration (Figs. 4A, B) and invasion (Figs. 4C, D) of both HCT15 and SW620 cells.
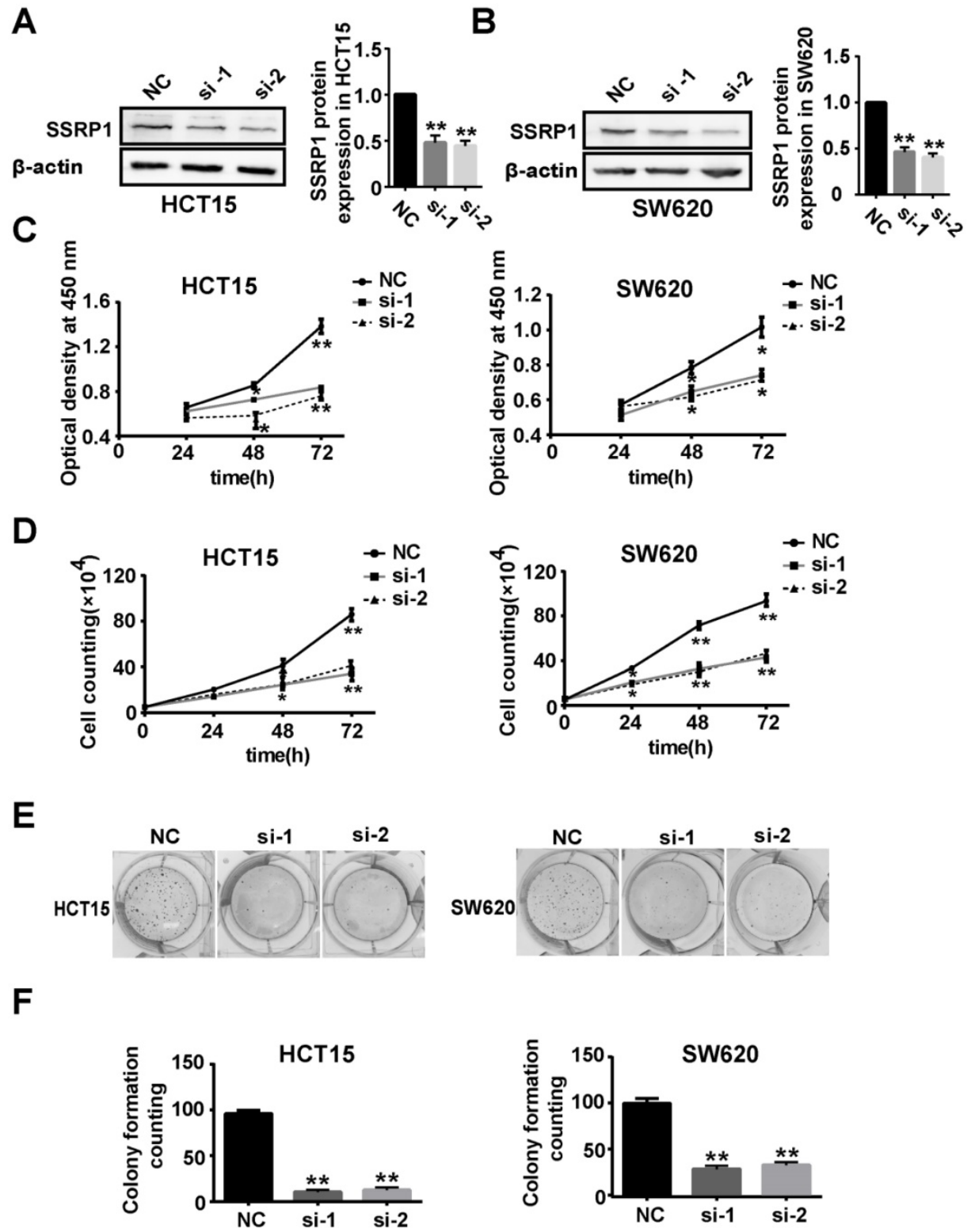

Figure 2. SSRPI inhibition represses proliferation of colorectal cancer cells. (A, B) Protein levels of SSRPI in HCT15 and SW620 cells at 48 h post siRNA transfection and densitometric quantification of proteins normalized to $\beta$-actin. (C) CCK8 assay after siRNA transfection for $48 \mathrm{~h}$ at OD $450 \mathrm{~nm}$ in HCT15 and SW620 cells. (D) The growth curve of HCT15 and SW620 cells after siRNA transfection. (E, F) Colony formation ability of HCT15 and SW620 cells after SSRP1 silence by transfecting siRNA. $* P<0.05, * * P<0.01$ vs NC group. 
A
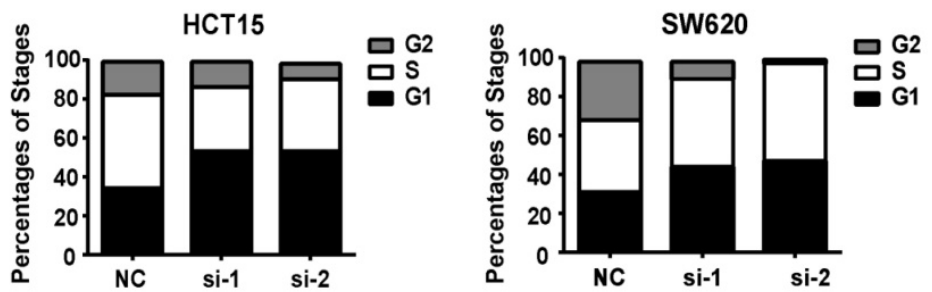

B
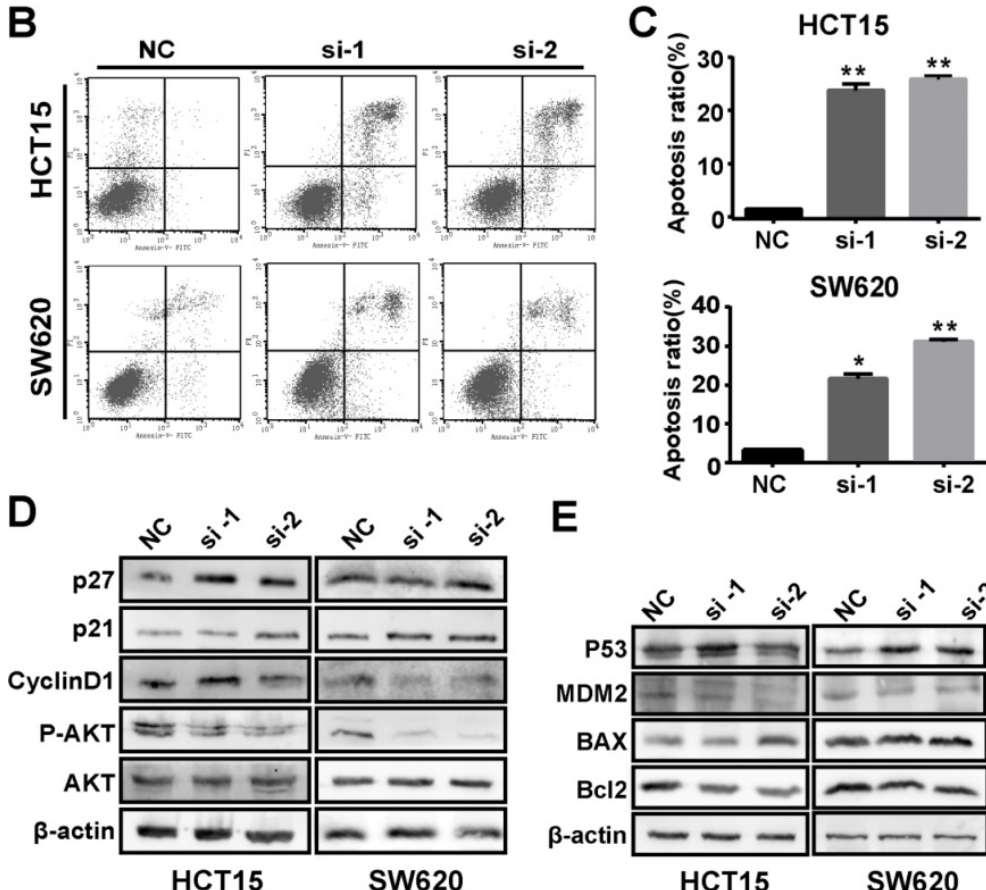

E

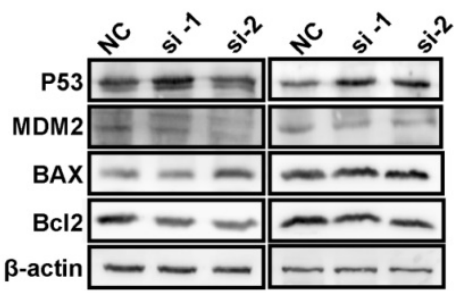

$\mathbf{F}$

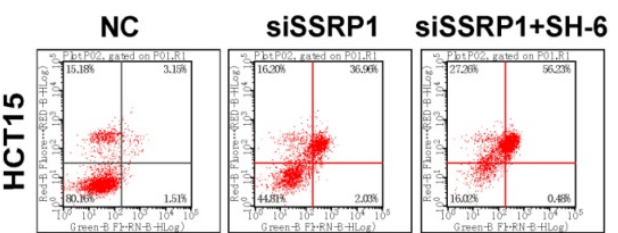

$\mathrm{H}$

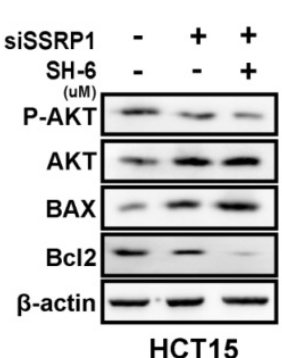

I

HCT15 SW620

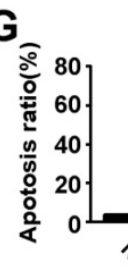

HCT15
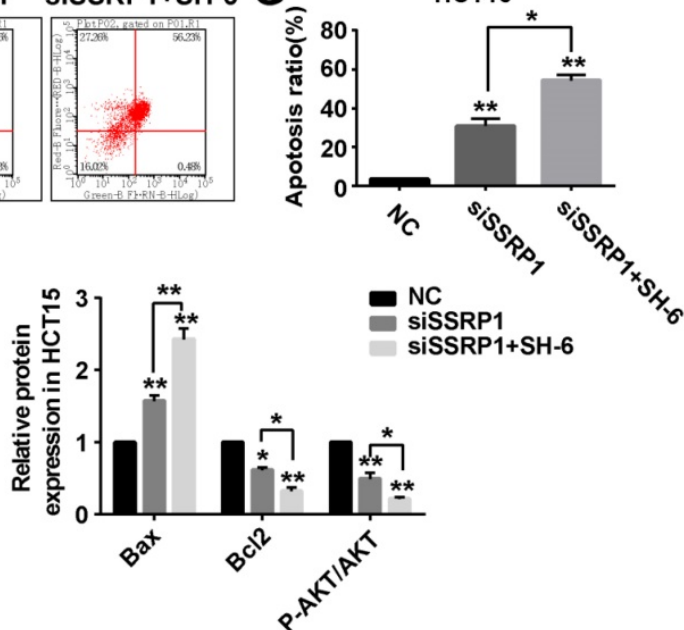

Figure 3. SSRP I exerts proliferation and apoptosis of colorectal cancer cells by activating the AKT pathway. (A) Graphs with quantitative data for the flow cytometric cell cycle distribution assay in HCT15 and SW620 cells. (B, C) FITC Annexin V/PI staining indicated increased apoptosis in HCT15 and SW620 cells with SSRP1 inhibiting. (D, E) Western blot displayed reduced P-AKT, CyclinD1, BCL-2 and MDM2, accompanying increased p27, p21, p53 and BAX in transfected HCT15 and SW620 cells. $(\mathbf{F}, \mathbf{G})$ Apoptosis ratio in HCT15 cells with SSRP1 or AKT inhibitors through flow cytometry. $(\mathbf{H}, \mathbf{I})$ The protein level of P-AKT, AKT, BAX and BCL-2 in HCT15 cells with SSRPI or AKT inhibitors. $* P<0.05, * * P<0.01$ vs NC group.

\section{SSRPI inhibition blocked colorectal cancer growth in vivo}

Since SSRP1 knockdown altered the proliferation and apoptosis of colorectal cancer in vitro as well as the migration and invasion of colorectal cancer cells, we attempted to determine whether the same phenomenon would occur in vivo. We used the HCT15 cell line to construct a colorectal cancer xenograft model and randomly divided nude mice 
into the siSSRP1 and NC groups. When the tumor size reached $3-5 \mathrm{~mm}$, either siSSRP1 $(0.5 \mathrm{mg} / \mathrm{kg})$ or NC was inoculated into xenograft tumors by multipoint injection three times per week (Fig. 5A). The siSSRP1 group exhibited a significantly smaller tumor volume and growth rate (Fig. 5B, C); however, no significant differences occurred in body weight between the two groups (Supplementary Fig. S3A). We also verified the expression of SSRP1 by western blotting and immunohistochemistry. The expression of SSRP1 in the siSSRP1 group was markedly downregulated (Fig. $5 \mathrm{D}, \mathrm{E})$. TUNEL assay was performed to detect the apoptotic cells, which demonstrated that the siSSRP1 group had strong green fluorescence intensity at the same exposure time (Fig. 5F). This indicated that that SSPR1 silencing induced apoptosis in the colorectal cancer cells in vivo. Moreover, the results of immunohistochemistry also confirmed that downregulation of SSRP1 could undermine the expression of PCNA, BCL-2, MMP-2 and MMP-9, and at the same time, it also induced the an increase in the expression of BAX (Fig. 5G). Consistent with the in vitro results, SSRP1 knockdown in vivo activated the AKT signaling pathway and altered the expression of downstream proteins related to the cell cycle and apoptosis. Collectively, these data showed that downregulation of SSRP1 inhibited colorectal cancer progression in vivo.

A

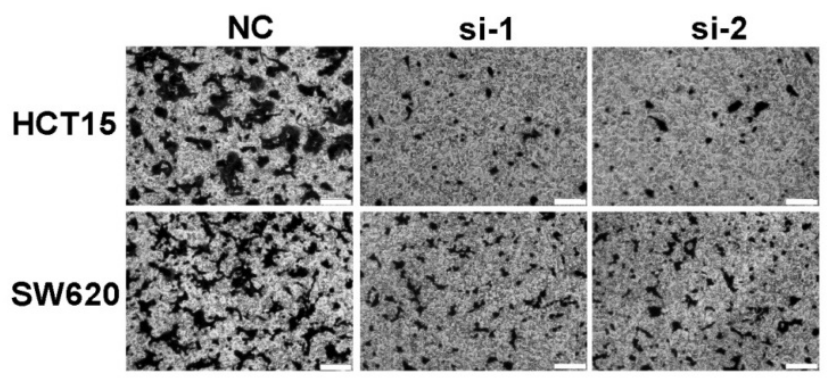

B

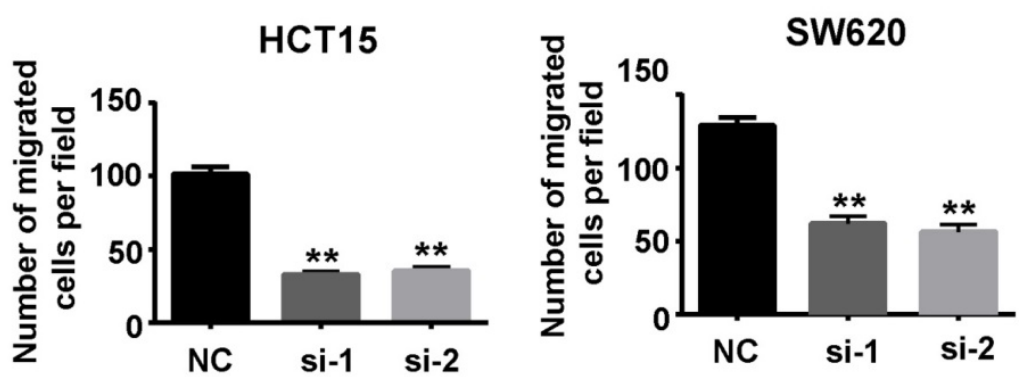

C

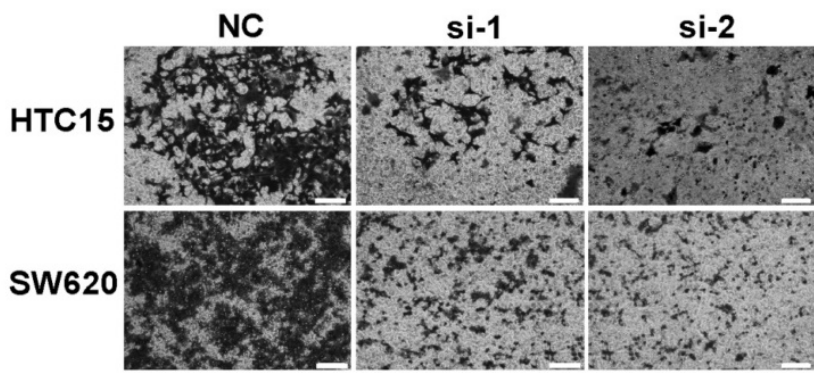

D
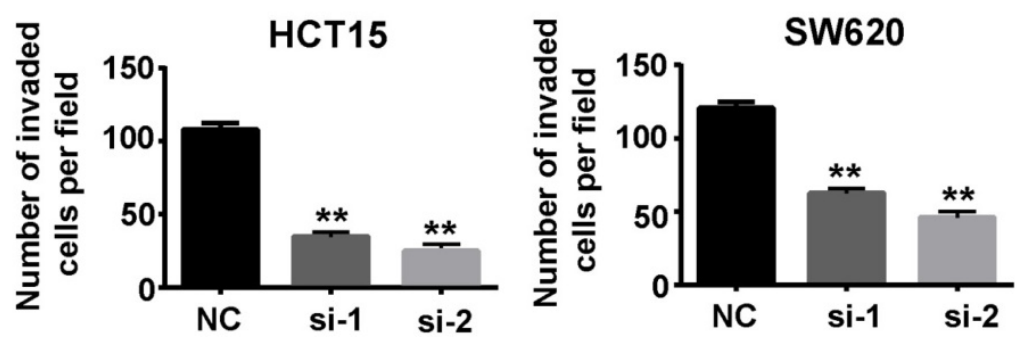

Figure 4. Downregulation of SSRPI inhibits migration and invasion of colorectal cancer cells. Migration (A, B) and invasion (C, D) ability of HCT15 and SW620 cells through transwell assay after SSRPI downregulation. $* P<0.05$, $* * P<0.01$ vs NC group. 
A

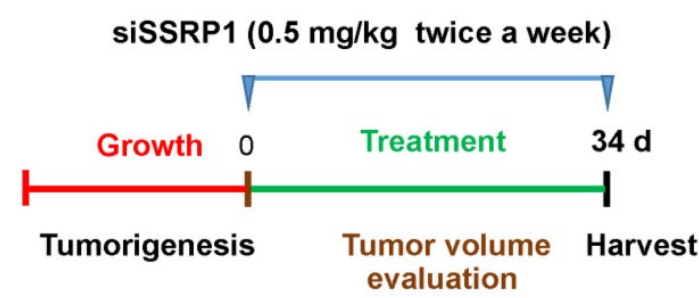

C

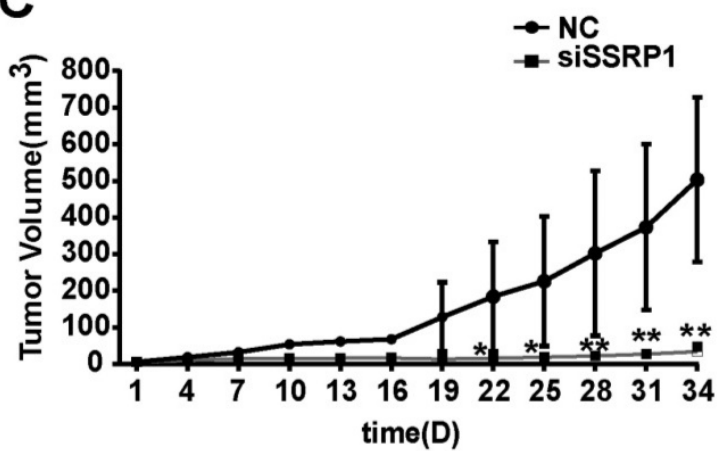

B

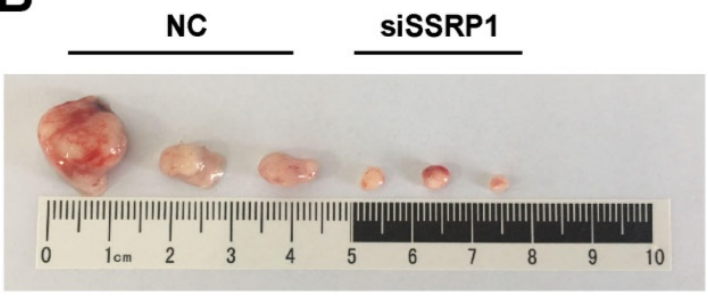

D

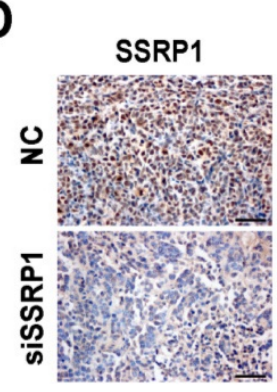

E

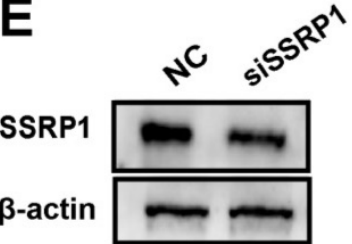

$\mathbf{F}$

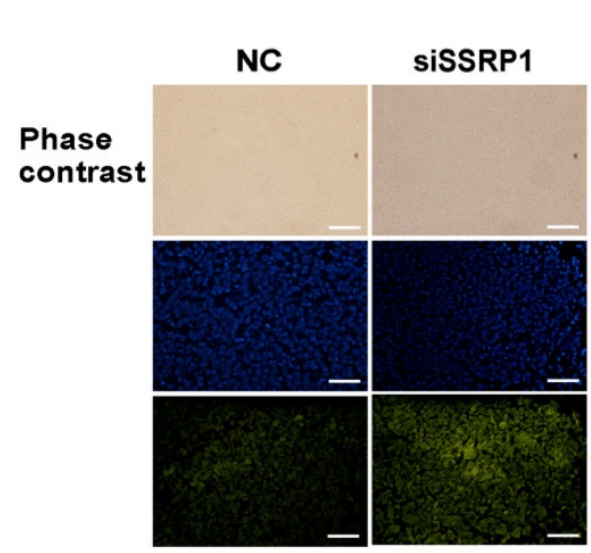

$\mathbf{H}$
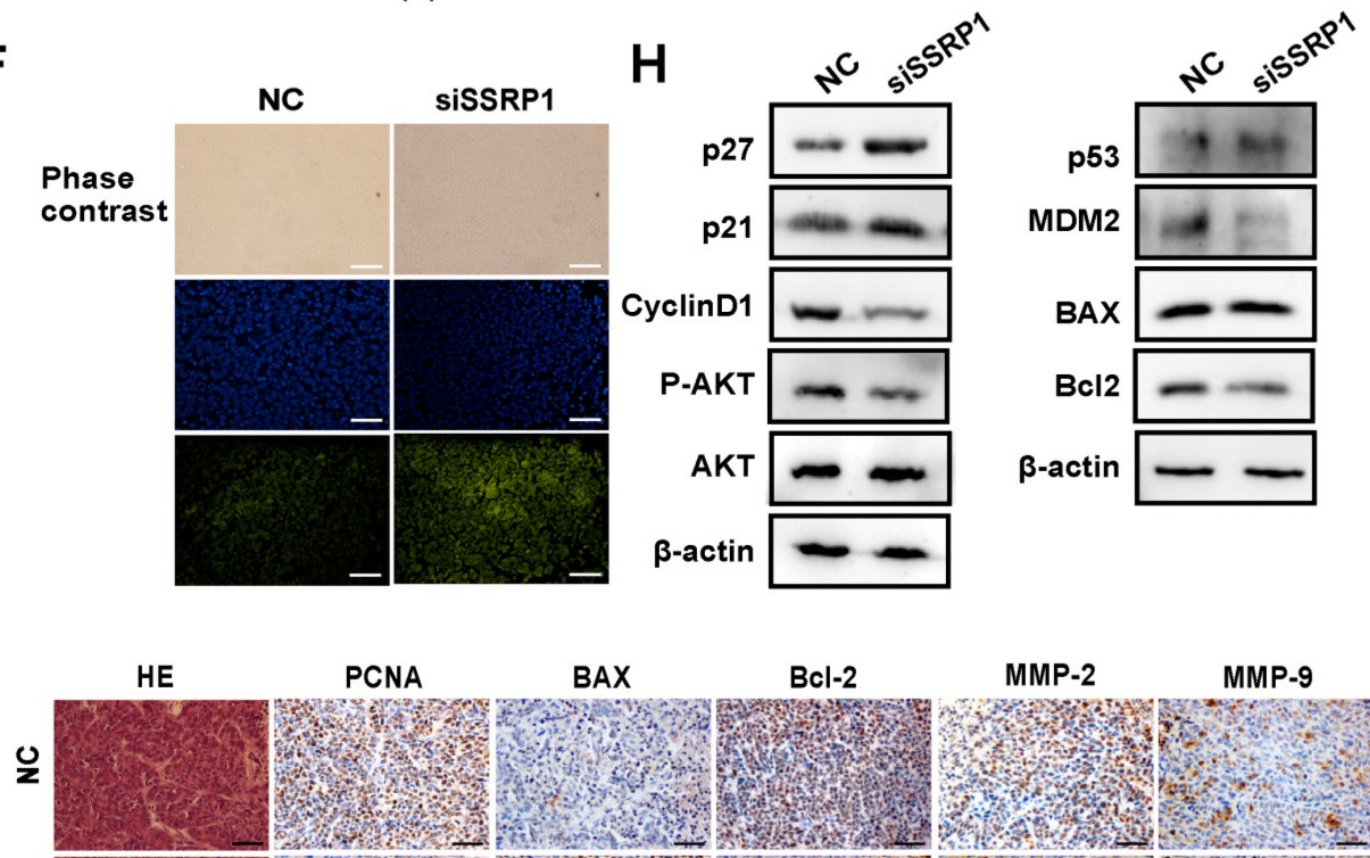

BAX

$\mathrm{Bcl}-2$

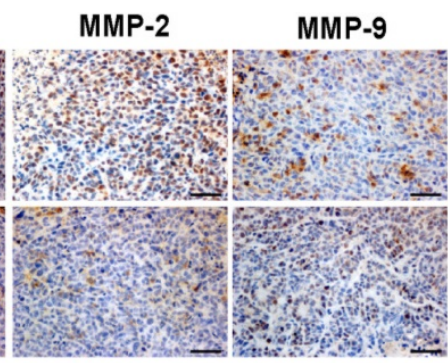

Figure 5. SSRPI inhibition blocks the growth of colorectal cancer in vivo. (A) Treatment for transplanted xenogeneic models using HCT15 cells. (B) Image of the tumor tissues of NC group and siSSRP1 group. (C) Tumor growth curve of NC group and siSSRPI group for $34 \mathrm{~d}$ treatment. (D) Immunofluorescent staining showing that SSRPI expression was reduced in siSSRPI group, compared with NC group. (E) Validation of SSPRI protein levels through western blot. (F) TUNEL assay of tumor tissue. (G) Immunofluorescent staining of PCNA, BAX, Bcl2, MMP-2 and MMP-9. (H) AKT, P-AKT, p27, p21, CyllinD1, p53, MDM2, BAX, and Bcl2 protein levels were determined by western blot analysis. $* P<0.05, * * P<0.01$ vs $N C$ group.

\section{Discussion}

The histone chaperone protein, FACT, is composed of SSRP1 and SUPT16H (suppressor of Ty 16 homolog) and assists in unraveling DNA molecules from histone octamers, reduces the compactness of nucleosomes, and promotes extension during transcription 9,10 . It is also closely related to cell proliferation and apoptosis ${ }^{11}$. Current research on SSRP1 indicates that it is upregulated in various tumor types, ${ }^{5}$ 12. Moreover, changes in SSRP1 expression affect tumor characteristics; for example, 
curaxins, inhibitory compounds with specific activity against FACT, induce apoptosis in tumor cells by activating p53 and inhibiting NF-KB ${ }^{13}$. Downregulation of SSRP1 expression by siRNA interference also results in the proliferation of U87 and U251 glioma cells through the MAPK pathway ${ }^{8}$. In addition, lower FACT expression has been reported in more differentiated cells compared with stem cells, progenitor cells, and less differentiated cells, and expression levels changed after experimentally inducing differentiation ${ }^{14}$. Our findings and those of previous studies suggest that SSRP1 may be a target gene for cancer therapy, but further work is required to determine the potential function of SSRP1 in colorectal cancer progression.

Colorectal cancer can be divided into three histological types: adenocarcinoma, mucinous carcinoma, and undifferentiated carcinoma. Colorectal adenocarcinoma accounts for approximately three-quarters of colorectal cancer cases. Our analysis of data from TCGA confirmed that SSRP1 is upregulated in many human tumor tissues, including colorectal adenocarcinoma, along with multiple colorectal cancer cell lines. We also found that, compared with normal patients, colorectal adenocarcinoma patients showed significantly increased SSRP1 expression levels, as demonstrated by histochemical staining results from the Human Protein Atlas database. This indicated that SSRP1 may act as an oncogene in the development of colorectal cancer. To verify this, we downregulated the SSRP1 expression using siRNA and found that SSRP1 inhibition significantly inhibited proliferation and metastasis and promoted apoptosis of colorectal cancer in vivo and in vitro. However, the small number of tumor tissues was still a shortcoming in our research process.

The PI3K-AKT signaling pathway is a classic signaling pathway associated with cell survival and apoptosis, and the serine/threonine kinase, AKT (also known as protein kinase B), which comprises a group of three isoforms (AKT1, AKT2, and AKT3) in mammals, is a critical propagator of PI3K signaling 15,16 .

Activated AKT phosphorylates many substrates controlling almost every aspect of various physiological and pathological cellular functions, including cell survival, growth, metabolism, tumorigenesis, and metastasis ${ }^{17-19}$. We thus investigated whether the AKT signaling pathway participated in the changes in proliferation and apoptosis in colorectal cancer after SSRP1 intervention. Our results revealed that SSRP1 silencing activated the AKT signaling pathway to regulate proliferation, metastasis, and apoptosis in colorectal cancer.
However, further research to clarify the molecular mechanism of the interaction between SSRP1 and AKT pathway. In summary, the present study confirmed that SSRP1 influences the proliferation and apoptosis of colorectal cancer cells via the AKT pathway.

\section{Abbreviations}

SSRP1: structure-specific recognition protein 1; TCGA: the Cancer Genome Atlas.

\section{Supplementary Material}

Supplementary figures.

http://www.medsci.org/v16p1573s1.pdf

\section{Acknowledgements}

The present study was funded by the National Natural Science Foundation of China (grant no. 81773217), International Cooperation Project of Jilin Provincial Science and Technology Department (grant no.20190701065GH), Jilin University Bethune Plan B Projects (No. 2015220) and Graduate Innovation Fund of Jilin University (No. 101832018c058).

\section{Authors' contributions}

Conception and design: QW, SNJ and YJ; Development of methodology: QW, BFG and LZ; Acquisition of data: SNJ and YJ; Analysis and interpretation of data: QW, SNJ, YJ and NYW; Writing, review and/or revision of the manuscript: QW, BFG and LZ; Administrative, technical, or material support: QW, BFG and LZ; Study supervision: BFG and LZ; Other: $\mathrm{XYC}, \mathrm{DW}, \mathrm{XDH}$, LBX, HL and SNZ.

\section{Competing Interests}

The authors have declared that no competing interest exists.

\section{References}

1. Chen W, Zheng R, Baade PD, et al. Cancer statistics in China, 2015. CA Cancer J Clin. 2016;66(2):115-132.

2. Zeng M, Zhu L, Li L, Kang C. miR-378 suppresses the proliferation, migration and invasion of colon cancer cells by inhibiting SDAD1. Cell Mol Biol Lett. 2017;22:12.

3. Wang X, Liu J, Jiang L, et al. Bach1 Induces Endothelial Cell Apoptosis and Cell-Cycle Arrest through ROS Generation. Oxid Med Cell Longev. 2016;2016:6234043

4. Davudian S, Mansoori B, Shajari N, Mohammadi A, Baradaran B. BACH1, the master regulator gene: A novel candidate target for cancer therapy. Gene. 2016;588(1):30-37.

5. Ding $\mathrm{Q}, \mathrm{He} \mathrm{K}$, Luo $\mathrm{T}$, et al. SSRP1 Contributes to the Malignancy of Hepatocellular Carcinoma and Is Negatively Regulated by miR-497. Mol Ther. 2016;24(5):903-914.

6. Huang H, Santoso N, Power D, et al. FACT Proteins, SUPT16H and SSRP1, Are Transcriptional Suppressors of HIV-1 and HTLV-1 That Facilitate Viral Latency. J Biol Chem. 2015;290(45):27297-27310.

7. Koessler J, Trulley VN, Bosch A, et al. The role of agonist-induced activation and inhibition for the regulation of purinergic receptor expression in human platelets. Thromb Res. 2018;168:40-46.

8. Liao J, Tao X, Ding Q, et al. SSRP1 silencing inhibits the proliferation and malignancy of human glioma cells via the MAPK signaling pathway. Oncol Rep. 2017;38(5):2667-2676. 
9. Erkina TY, Erkine A. ASF1 and the SWI/SNF complex interact functionally during nucleosome displacement, while FACT is required for nucleosome reassembly at yeast heat shock gene promoters during sustained stress. Cell Stress Chaperones. 2015;20(2):355-369.

10. Tsunaka Y, Fujiwara Y, Oyama T, Hirose S, Morikawa K. Integrated molecular mechanism directing nucleosome reorganization by human FACT. Genes Dev. 2016;30(6):673-686

11. Gao XJ, Feng JX, Zhu S, Liu XH, Tardieux I, Liu LX. Protein Phosphatase 2C of Toxoplasma Gondii Interacts with Human SSRP1 and Negatively Regulates Cell Apoptosis. Biomed Environ Sci. 2014;27(11):883-893.

12. Garcia H, Miecznikowski JC, Safina A, et al. Facilitates chromatin transcription complex is an "accelerator" of tumor transformation and potential marker and target of aggressive cancers. Cell Rep. 2013;4(1):159-173.

13. Gasparian AV, Burkhart CA, Purmal AA, et al. Curaxins: anticancer compounds that simultaneously suppress NF-kappaB and activate p53 by targeting FACT. Sci Transl Med. 2011;3(95):95ra74.

14. Garcia H, Fleyshman D, Kolesnikova K, et al. Expression of FACT in mammalian tissues suggests its role in maintaining of undifferentiated state of cells. Oncotarget. 2011;2(10):783-796.

15. Mabuchi S, Kuroda H, Takahashi R, Sasano T. The PI3K/AKT/mTOR pathway as a therapeutic target in ovarian cancer. Gynecol Oncol. 2015;137(1):173-179.

16. Laplante M, Sabatini DM. mTOR signaling in growth control and disease. Cell. 2012;149(2):274-293.

17. Mabuchi S, Hisamatsu T, Kimura T. Targeting mTOR signaling pathway in ovarian cancer. Curr Med Chem. 2011;18(19):2960-2968.

18. Guo H, German P, Bai S, et al. The PI3K/AKT Pathway and Renal Cell Carcinoma. J Genet Genomics. 2015;42(7):343-353.

19. Dai C, Xie Y, Zhuang X, Yuan Z. MiR-206 inhibits epithelial ovarian cancer cells growth and invasion via blocking c-Met/AKT/mTOR signaling pathway. Biomed Pharmacother. 2018;104:763-770. 